\title{
The Stardust Comet Sample Return Mission and the Application of Microbeam Analyses on Small Particles.
}

\author{
D. E. Brownlee, Department of Astronomy, University of Washington, Seattle,WA 98195
}

Stardust is a NASA mission designed to collect particulate samples from a comet and return them to Earth. As a sample return mission its major science return will come from laboratory studies of many investigators around the world using the best available analytical methods [1]. The samples were collected on January 2, 2004 during a close flyby of comet Wild 2 and they are now heading home for a landing in Utah on January 15 2006. Thousands of particles ranging in size from a few microns to over $100 \mu \mathrm{m}$ in diameter were collected by the $6.1 \mathrm{~km} \mathrm{~s}^{-1}$ impact of comet dust into silica aerogel, a micro-porous silica with graded densities ranging from $0.005 \mathrm{~g} \mathrm{~cm}^{-3}$ to $0.05 \mathrm{~g} \mathrm{~cm}^{-3}$. The samples, collected from the dust cloud surrounding Wild 2, were released from the comet due the gentle sublimation of ice and other volatiles that had bonded the comet together since its formation 4.6 billion years ago near the orbit of Pluto. The collected particles are believed to be representative samples of the fundamental building materials that began the planet formation process in the outer solar system. They are expected to be preserved samples of materials that formed in the solar nebular disk as well as interstellar grains that formed around other stars and resided for a substantial period of time in the interstellar medium. The returned samples will provide a remarkable opportunity to compare materials that formed bodies beyond the orbit of Neptune with materials preserved in asteroidal meteorites that formed in the inner solar system just beyond the orbit of Mars. Stardust is the first NASA sample return mission launched since Apollo 17 in 1972 and the first ever sample return mission beyond the Moon.

The Stardust sample collection will consist of many thousands of particles imbedded in lowdensity silica aerogel. Particles that do not fragment during collection will produce carrot-shaped tracks in the aerogel with the particle at the tip of the cone. For non-fragmenting materials, a $10 \mu \mathrm{m}$ particle can produce a track over a millimeter long. Particles can be extracted from the aerogel capture medium using a variety of methods including computer driven needles that extract wedge shaped aerogel samples [2] to methods that use vibrating diamond knifes [3]. The samples will be curated at the NASA Johnson Space Center in Houston Texas and they will be distributed to interested scientists using an allocation system based on those used for distribution of Lunar samples, cosmic dust and Antarctic Meteorites.

Two of initial goals of the comet particle study will be: A) to determine if the comet samples are similar to or distinct from known types of materials found in interplanetary dust or meteorites and B) to estimate the fraction of particles in the comet are presolar grains that predate the solar system. Like other primitive meteoritic materials it is expected that the comet particles will be very fine grained (composed of submicron grains), black and have typical bulk elemental compositions that are similar to those of primitive undifferentiated meteorites- basically matching those of the Sun for rock forming elements.

Most of the returned samples will be in the $5 \mu \mathrm{m}$ to $25 \mu \mathrm{m}$ size range and the analytical techniques used on them will be similar to those used to study interplanetary dust (IDPs) samples of the same size. Over the years the ability to work on these types of cosmic particles has advanced in remarkable ways [4-6]. In terms of particle analysis, the study of IDPs has peculiar challenges. 
Many of the particles are highly inhomogeneous and a typical $10 \mu \mathrm{m}$ diameter particle is actually an aggregate, a collection, of tens of thousands of smaller particles each of which has its own history and story to tell. Over a span of a micron or so you might find a silicate grain, that formed around another star, organic material that may have formed in the solar system and a magnetite grain that may have formed during recent pulse heating of the particle. Because of the complexity each $10 \mu \mathrm{m}$ particle is actually a unique rock with its own history and it must be treated accordingly. Each particle is precious and as many analytical processes as possible must be used on the same sample thus providing challenging analysis schemes designed to maximize the information yield.

All modern work on nanogram IDPs relies heavily on enabling technologies developed over the last few decades. When interplanetary dust samples were first collected in 1970 (first using high altitude balloons and then U2 airplanes) their identification and distinction from contaminants and other stratospheric particles was possible because of the newly developed techniques of SEM-EDX. The most common primitive meteoritic particles have compositions similar to that of the Sun (for $\mathrm{Na}$, $\mathrm{Mg}, \mathrm{Al}, \mathrm{Si}, \mathrm{S}, \mathrm{Ca}, \mathrm{Cr}, \mathrm{Mn}, \mathrm{Fe}$ and $\mathrm{Ni}$ ) and this allowed them to be distinguished from essentially all common contaminants and natural atmospheric particulates. SEM-EDX is a powerful means of doing routine examination of these types of particles and with special effort these methods can be used to provide quantitative elemental analyses, particle masses and densities. Unfortunately SEMEDX is not a good means of studying the submicron components of typical IDPs. A critical enabling technology in this field was the development of microtomy techniques that could slice these $10 \mu \mathrm{m}$ rocks into hundreds of 100nm sections [7]. Recent efforts using Focused Ion Beam methods are an important extension of "conventional microtomy". Microtome sections provide a means of TEM study, with all its analytical capabilities, of the samples down to atomic resolution and provide a fabulous means to examine the thousands of submicron components in typical $10 \mu \mathrm{m}$ IDPS. The microtomy of these samples has advanced by the use of sulfur and acrylic imbedding media to produce sections that retain no carbon from the microtomy process [8]. Organic carbon is a major component of IDPs and comets and its study is a major research goal. Microtome slices have also proved to be suitable for isotopic study using modern secondary ion microprobes (SIMS) with submicron beam sizes. Combined TEM and SIMS can be done either on the same slice or on adjacent serial slices, providing that ability to do isotopic studies on submicron components and do full analytical spectroscopy. It is hoped that all of the combined abilities of analytical microscopy, SIMS analysis and other methods such as accelerator and synchrotron microbeam techniques will provide an intimate view of the elemental, mineralogical and isotopic nature of a comet that has preserves the initial building blocks or our solar system.

[1] D. E. Brownlee et al., Journal of Geophysical Research (Planets) 108 (2003) 1-1.

[2] A. J. Westphal et al., Meteoritics and Planetary Science 37 (2002) 855.

[3] J.P. Bradley et al, Lunar and Planetary Sci. Conf. (2005) in press.

[4] J.P. Bradley, Interplanetary Dust Particles, in Treatise of Geochemistry Vol 1 Ed A. Davis Elsiever (2004).

[5] E. Jessberger, et al., Properties of interplanetary dust: information from collected samples, in Interplanetary Dust, E. Grun, et al., Eds Springer, New York, (2001) 253.

[6] F. Rietmeijer, Interplanetary Dust Particles, in Reviews of Mineralogy 36 ( 1999) 95.

[7] J. P Bradley and D. E. Brownlee, Science 231 (1986) 1542.

[8] J. P Bradley et al., Lunar and Planetary Institute Conference Abstracts 24 (1993)173. 